\title{
A Chronological Atlas of Natural Selection in the Human Genome during the Past Half-million Years
}

Hang Zhou ${ }^{1,2,10}$, Sile $\mathrm{Hu}^{1,2,10}$, Rostislav Matveev ${ }^{3,4}$, Qianhui $\mathrm{Yu}^{1,2}$, Jing $\mathrm{Li}^{1}$, Philipp

Khaitovich $^{1}$, Li Jin ${ }^{1,5}$, Michael Lachmann ${ }^{3,6}$, Mark Stoneking ${ }^{3}$, Qiaomei Fu ${ }^{7,8,9}$ \& Kun Tang ${ }^{1}$

${ }^{1}$ Key Laboratory of Computational Biology, CAS-MPG Partner Institute for Computational Biology, Shanghai Institutes for Biological Sciences, Chinese Academy of Science, Shanghai 200031, China.

${ }^{2}$ University of Chinese Academy of Sceiences, Beijing 100049, China.

${ }^{3}$ Department of Evolutionary Genetics, Max Planck Institute for Evolutionary Anthropology, D-04103 Leipzig, Germany.

${ }^{4}$ Max Planck Institute for Mathematics in the Sciences, D-04103 Leipzig, Germany.

${ }^{5}$ MOE Key Laboratory of Contemporary Anthropology, Fudan University, Shanghai 200433, China.

${ }^{6}$ Santa Fe Institute, Santa Fe, New Mexico 87501, USA.

${ }^{7}$ Department of Genetics, Harvard Medical School, Boston, Massachusetts 02115, USA.

${ }^{8}$ Broad Institute of Harvard and MIT, Cambridge, Massachusetts 02142, USA

${ }^{9}$ Key Laboratory of Vertebrate Evolution and Human Origins of Chinese Academy of Sciences, IVPP, CAS, Beijing 100044, China.

${ }^{10}$ These authors contributed equally to this work.

Correspondence could be addressed to K.T. (tangkun@picb.ac.cn) 
The spatiotemporal distribution of recent human adaptation is a long standing question. We developed a new coalescent-based method that collectively assigned human genome regions to modes of neutrality or to positive, negative, or balancing selection. Most importantly, the selection times were estimated for all positive selection signals, which ranged over the last half million years, penetrating the emergence of anatomically modern human (AMH). These selection time estimates were further supported by analyses of the genome sequences from three ancient AMHs and the Neanderthals. A series of brain function-related genes were found to carry signals of ancient selective sweeps, which may have defined the evolution of cognitive abilities either before Neanderthal divergence or during the emergence of AMH. Particularly, signals of brain evolution in AMH are strongly related to Alzheimer's disease pathways. In conclusion, this study reports a chronological atlas of natural selection in Human. 
The extent to which human evolution has been influenced by natural selection is a contentious issue. Key transition periods of strong evolutionary importance include the divergence from archaic hominins such as Neanderthals and Denisovans, the emergence of anatomically modern humans (AMH), migration out of Africa (OOA), and agricultural expansions. The last decade has seen great progress in identifying the genomic signals of positive selection (PS $)^{1-8}$. Causal links to specific selection events have been established for a dozen candidate loci , including LCT , SLC24A5, KITLG, $A M Y 1, E D A R$ and EGLN1 supported by evidence from functional assays ${ }^{9-14}$. Furthermore, efforts have been made to estimate the starting time of PS events. Inference of selection time helps assign a PS signal to a specific evolutionary context and is critical to the understanding of the evolutionary roles the underlying genetic changes played. Itan et al. ${ }^{15}$ estimated the selection on $L C T$ to be around 7,500 years ago (ya), based on an approximate Bayesian computation (ABC) based approach . Beleza et al. ${ }^{16}$ used a forward Monte Carlo simulation coupled with a rejection sampling, and estimated the selection starting times for several genes of pigmentation lightening to be within the last 11,000-30,000 years in Europeans . In East Asia, the selection sweep in EDAR was estimated to have occurred around 30,000 ya, based on $\mathrm{ABC}$ inference ${ }^{13}$. Recently, the advance in methods of ancient DNA re-sequencing made it possible to examine the allele frequency trajectory of putative PS loci along real time, thus lending direct supports to the PS events and the corresponding selection time ${ }^{17,18}$. However, selection time estimation was restricted to a few wellstudied cases, mainly due to the high computational cost and lack of generalized methods. Furthermore, the latest powerful tests based on extended haplotypes were found to be mainly sensitive to very recent events, e.g. not beyond $30,000 \mathrm{ya}^{19}$. It was argued that PS events older than 250,000 ya might not leave detectable signatures given the conventional test frameworks ${ }^{20,21}$. Finally, soft sweep, a mode of positive selection where selection acts on multiple standing variants, also hinders a comprehensive understanding of the past PS events due to low test power. Some newly developed methods showed improved test power towards soft sweeps ${ }^{2,22,23}$.

Compared to PS, much less work has been conducted on negative selection $(\mathrm{NS})^{24,25}$ or balancing selection $(\mathrm{BS})^{26-28}$. Approximately $5 \%-15 \%$ of the human 
genome is estimated to have been affected by $\mathrm{NS}^{29-31}$ and one recent genome scan identified a few hundred candidate regions of $\mathrm{BS}^{32}$.

In the last few years, a new class of methods has emerged, which base the population genetic inference on reconstructed coalescent information ${ }^{23,33,34}$. These methods manifested promising test power and resolution for inferring selection, as the genome-wide coalescent trees provide a complete record of genealogies, mutations, and recombination ${ }^{33}$. Still, while genome scans provide lists of candidate loci, identifying the actual targets of selection and determining when, how, and why selection has acted on a particular target remain daunting tasks. In this study, we present a coalescent model-based method that simultaneously identifies genome-wide signals of PS, NS, and BS (as well as neutrality). Coalescent patterns associated with PS signals were further queried for selection starting times and selection coefficients.

\section{RESULTS}

\section{The model}

Our method starts with reconstructing the coalescent trees from genome sequence data by using the pairwise sequentially Markovian coalescent (PSMC) model ${ }^{35}$. Thirty haploid genome sequences were randomly selected from each of the CEU, CHB, and YRI panels of the 1000 Genomes Project (1000G) phase 1 dataset $^{36}$. Within each population, a distance matrix of the time since the most recent common ancestor (TMRCA) was derived by applying PSMC to all pairs of haploid genomes and was used to estimate unweighted pair group method with arithmetic means (UPGMA) trees (raw trees) across the whole genome (Supplementary Fig. 1 and Online Methods). The use of $1000 \mathrm{G}$ phase 1 data was supported by comparison to highcoverage data (Supplementary Figs. 2 and 3, Supplementary Note 1.2). Simulations showed that the derived raw trees also approximate the local coalescent trees (Supplementary Fig. 4). The raw trees were consequently controlled for reconstruction errors and rescaled into a coalescent time scale, where effects of population size variation were eliminated (Online Methods). Rescaled neutral trees follow a standard constant-size coalescent distribution; therefore, various selection events can be detected as altered coalescent patterns. In this study, we based the detection of natural selection on the changing coalescent rates. In brief, NS/BS may 
be described by trees of globally enhanced/decreased coalescent rates. On the other hand, PS exhibits a sudden increase in the coalescent rate, the time of which should approximate the starting time of selection (Online Methods). We constructed a likelihood test framework to assign genomic regions to neutrality or different modes of selection. For PS, two tests were designed to be sensitive either toward recent selection events (hereafter referred to as the RPS test), or toward more ancient events (hereafter referred to as the APS test, Online Methods).

\section{Power test}

Simulations showed that our method has high power to detect NS and BS for a wide range of conditions (Supplementary Fig. 5). For the PS simulations based on realistic demographic models (Supplementary Fig. 6 and Online Methods), RPS and APS tests showed high combined power toward either recent $[<2,500$ generations ago (ga), Fig. 1a] or ancient (2,500-24,000 ga, Fig. 1b) events. Compared to several existing tests, including Tajima's $\mathrm{D}^{37}$, Fay and Wu's $\mathrm{H}^{38}$ and $\mathrm{iHS}^{2}$, only Tajima's D outperformed RPS and APS, and only around 1,000-1,500 ga (Fig. 1a,c). In the YRI scenario, the power of APS reached a maximum of $>90 \%$ around 2,500 ga and slowly declined to around $30 \%$ at time 20,000 ga (20 kga) (Fig. 1b). Following a commonly used consensus $^{35}$, we hereafter assume 25 years per generation; therefore, $20 \mathrm{kga}$ corresponds to 0.5 million years ago (mya). Interestingly, RPS and APS also have moderate power $(26 \%-87 \%)$ toward recent soft sweep events, clearly outperforming the other tests (Fig. 1d). Most importantly, our method simultaneously estimates selection coefficient and selection time. Simulations indicate that selection coefficients can best be estimated only roughly as strong or weak (Supplementary Fig. 7); however selection times could be estimated quite accurately (Fig. 1e-g and Supplementary Fig. 8). For recent selection events, the correlations between simulated and estimated selection times were $0.74\left(P=9.26 \times 10^{-124}\right), 0.52\left(P=7.09 \times 10^{-35}\right)$, and $0.71\left(P=6.53 \times 10^{-159}\right)$ for CEU, CHB, and YRI, respectively. Particularly in YRI, the correlation showed good linearity (Fig. 1f) persisted into the ancient time range (2,500-24,000 ga), although the correlation between the predicted and true values decreased slightly $\left(0.62, P=1.17 \times 10^{-137}\right.$, Fig. 1 g $)$. 
a

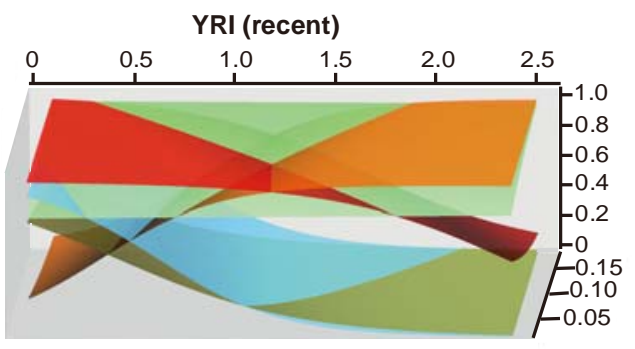

b

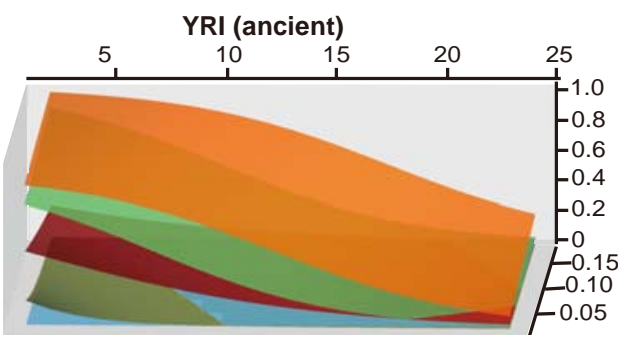

d

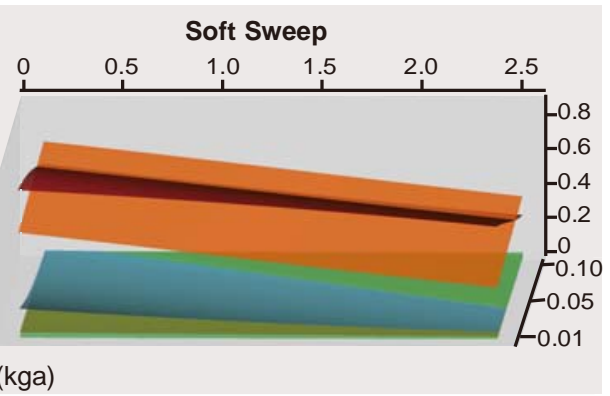

RPS

APS

iHS

Tajima's

D

Fay and

Wu's H e

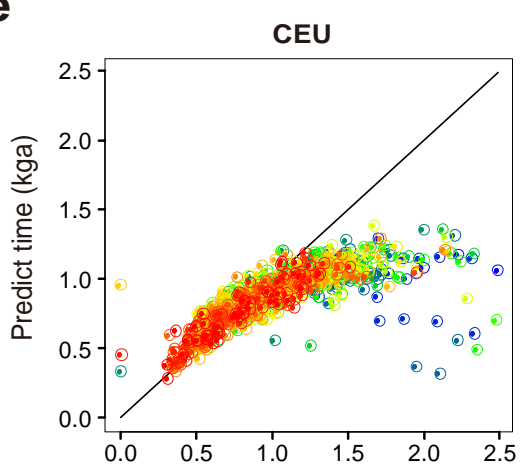

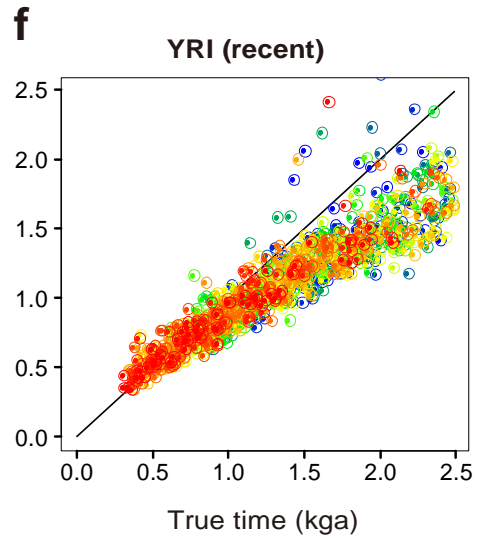

g

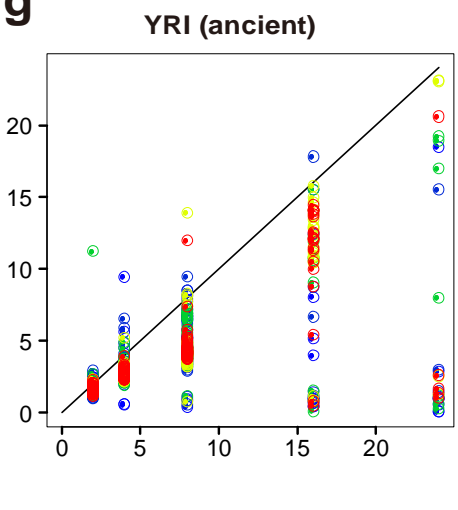

SC

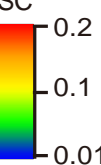

Figure 1 Power comparison and selection time estimation for PS. Test power of RPS $\left(D_{21}\right.$ recent) (red), APS ( $D_{21}$ ancient) (orange) were compared to that of Tajima's D (light green), iHS (light blue) and Fay and Wu's H (dark green) for hard sweep PS in YRI demography for (a) recent or (b) more ancient time ranges; or (c) in recent CEU demography. (d) Power comparison for soft sweeps in core demography. SC is selection coefficient, IF is initial allele frequency. Selection starting time was estimated for simulated PS in demographic scenarios for (e) recent CEU, (f) recent YRI, and (g) ancient YRI, respectively. The estimated times were plotted against the true selection starting times.

\section{Genome-wide signals of natural selection}


We identified 117, 230, and 485 candidate PS regions in CEU, CHB, and YRI, respectively (Fig. 2a,b and Supplementary Tables 1-3), which occupied 1.13\%-2.94\% of the genome. Functional enrichment analyses of the PS signals showed significant enrichment for genes expressed in the brain and sperm. In addition, in CEU and YRI enrichment was found for genes expressed in the pituitary gland, while enrichment for genes expressed in the appendix was observed in CHB and YRI. Other interesting categories include alcohol-metabolism in CHB and genes expressed in hair roots in CEU (Supplementary Tables 4-6 and Online Methods).

a

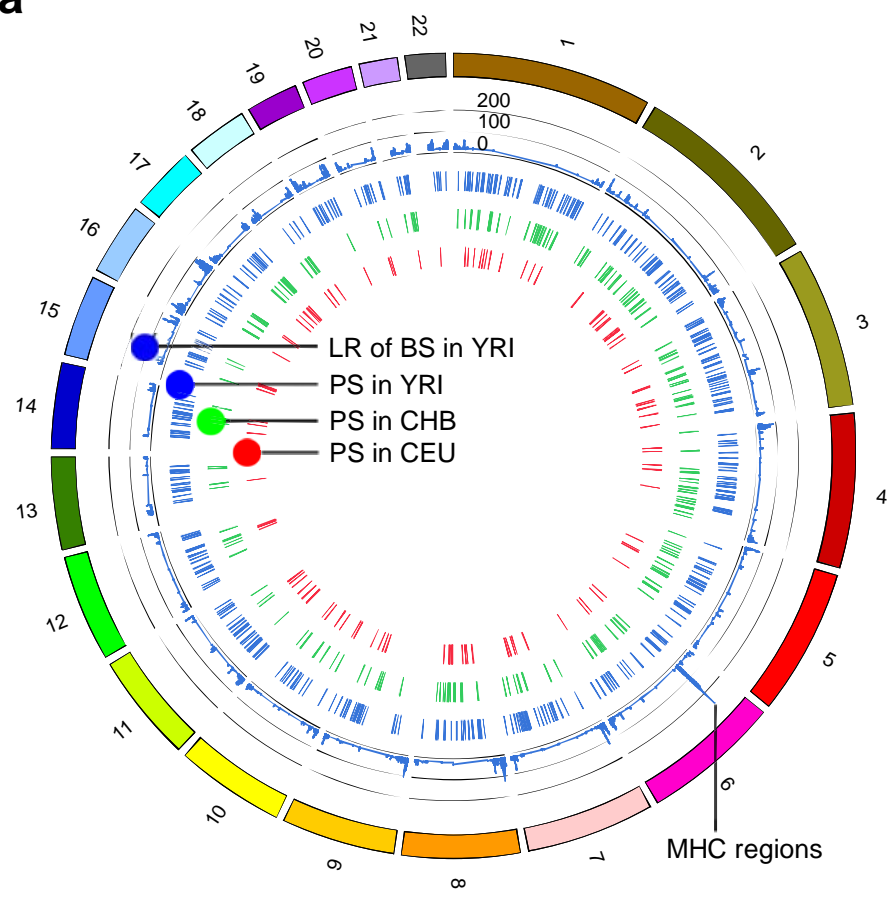

b

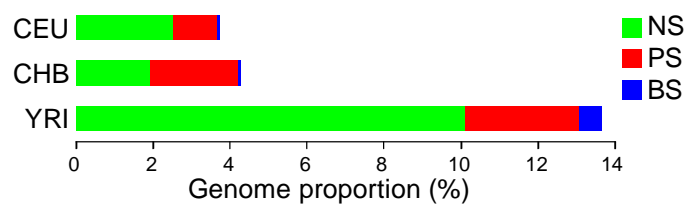

C

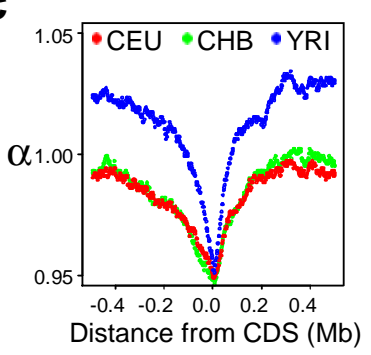

d

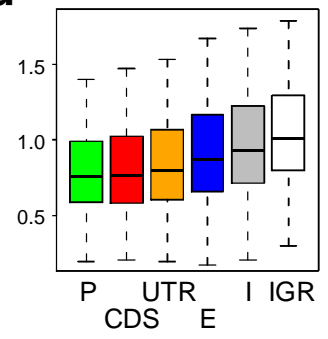

e

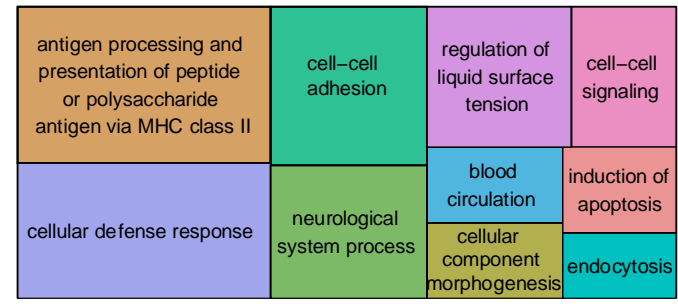

Figure 2 Genome-wide signals of natural selection. (a) The candidate regions of PS in CEU (red), CHB (green) and YRI (blue), and the $D_{10}$ likelihood ratio (LR) of BS signals in YRI. (b) Genome proportions of various types of natural selection estimated in CEU, CHB and YRI. (c) Genome average distribution of coalescent scaling coefficient $\alpha$ scores centered around the coding regions. (d) Distributions of $\alpha$ scores in different functional elements, including promoter regions (P), coding regions (CDS), untranslated regions (UTRs), enhancer regions (E), introns (I) and intergenic regions (IGR). (e) GO terms for biological processes significantly enriched for BS genes in YRI (see Supplementary Table 10 for details). The size of the rectangles reflects the $P$ value. 
Regarding the NS signals, a much higher genomic proportion of NS was observed in YRI $(\sim 10 \%)$ than in CEU $(\sim 2.5 \%)$ and CHB $(\sim 1.9 \%)$. One possible reason is that ancient signals of coalescent compression were eliminated by bottlenecks in nonAfrican populations. We used the tree scaling coefficient $\alpha$ to examine the effects of NS on different functional elements in YRI (Supplementary Note 4). $\alpha$ can be interpreted as the inverse coalescent rate compared to neutrality (Online Methods) and a clear decline of $\alpha$ was detected toward the centers of coding regions (CDS, Fig. 2c) and transcript start sites (TSS, Supplementary Fig. 9). The median $\alpha$ score was lowest in the promoter regions (0.755) and CDS (0.769), and progressively elevated in the untranslated regions (UTRs, 0.800), enhancers (0.875), introns (0.932), and intergenic regions (1.008), revealing a strong negative correlation with the expected functional essentiality of a genomic region (Fig. 2d). Patterns in CEU and CHB were similar to those observed in YRI (Supplementary Fig. 10).

Abundant signals of BS were detected in YRI, whereas much fewer BS signals were observed in CEU and CHB (Fig. 2a,b and Supplementary Tables 7-9). Notably, $71.3 \%$ and $62.2 \%$ of the BS signals in CEU and CHB were also annotated as BS in YRI. The major histocompatibility complex (MHC) regions harbored the strongest signals of BS in all three populations (Fig. 2a). Functional enrichment analyses of the BS signals revealed substantial involvement of multiple biological processes (Fig. 2e and Supplementary Tables 10-13, Online Methods), including: MHC class II ( $P=$ $3.67 \times 10^{-8}$, Bonferroni correction); cellular defense response $\left(P=3.86 \times 10^{-7}\right.$, Bonferroni correction); and cell-cell adhesion $\left(P=2.1 \times 10^{-5}\right.$, Bonferroni correction).

\section{Spatiotemporal distribution of positive selection}

If the genome-wide selection time estimation were accurate enough, it should cross-validate with ancient DNA (aDNA) evidence. Furthermore, the integration of both modern and aDNA evidence would provide a spatiotemporal roadmap of past adaptation events. In principle, if a selective sweep occurred prior to the time of an ancient specimen, then the aDNA from that specimen should harbor haplotypes derived from the beneficial haplotype which was fixed in the sweep (as should all of the other descendants in the same population). This predicts a small haplotype distance between the present-day sample and such aDNA sequences. It should be 
noted that an incomplete sweep or later outbreeding would disrupt such a relationship. On the other hand, if the ancient specimen dates to before the selective sweep, there is no explicit relationship between the aDNA and the present-day sequences, and therefore, their haplotype distance would vary across a large range. To test this, we used three ancient AMH genome sequences from previous studies: the 45,000 -yearold Ust'-Ishim man found in western Siberia (42× coverage) representing an ancient Eurasian $^{39}$ (hereafter referred to as aEA); a 7,000-year-old early European farmer, Stuttgart (aFM; 19x coverage); and a 8,000-year-old west European hunter-gatherer Loschbour $^{18}$ (aHG; $22 \times$ coverage). For a given candidate PS region, a measurement of haplotype distance $\left(D_{\mathrm{aDNA}, \mathrm{MHG}}\right)$ was calculated between the consensus sequence of the aDNA haplotypes and that of the major haplotype group (MHG) in the presentday sample (Online Methods). For the candidate PS regions, we observed a strong dependence of $D_{\mathrm{aDNA}, \mathrm{MHG}}$ on the estimated times of selection (Fig. 3). In CEU, the PS signals recorded a sudden decrease in the $D_{\mathrm{aDNA}, \mathrm{MHG}}$ distance to either aHG or aFM, along the estimated time of selection (Fig. 3a,b). The time that defines the most significant change in $D_{\text {aDNA,MHG }}$ (hereafter referred to as $T_{\max D}$ ) was estimated to be $835 \mathrm{ga}(20.9 \mathrm{kya})$ for aFM (Chi-square test, $P=1.0 \times 10^{-3}, \mathrm{FDR}_{\text {permut }}=0.029$, Fig. $3 \mathrm{a})$, and 706 ga $(\sim 17.7 \mathrm{kya})$ for aHG (Chi-square test, $P=2.44 \times 10^{-3}, \mathrm{FDR}_{\text {permut }}=$ 0.055 , Fig. 3b). These estimated times are substantially older than the radiocarbondated times of the aDNA specimens, potentially due to a tendency to overestimate the selection time in empirical CEU data. Alternatively, the early parting of the aDNA lineage from the direct ancestors of CEU or the strong population structure in the ancestral group, might also account for the observed time discrepancies. When YRI was compared to aEA, a similar decline of $D_{\mathrm{aDNA}, \mathrm{MHG}}$ was detected toward the more anciently dated signals, and $T_{\max D}$ was estimated to be 1,898 ga (47.5 kya, Chi-square test, $P=4.2 \times 10^{-5}, \mathrm{FDR}_{\text {permut }}=0.02$, Fig. $3 \mathrm{c}$ ), which was in good agreement with the results of radiocarbon dating for this specimen ${ }^{39}$. 


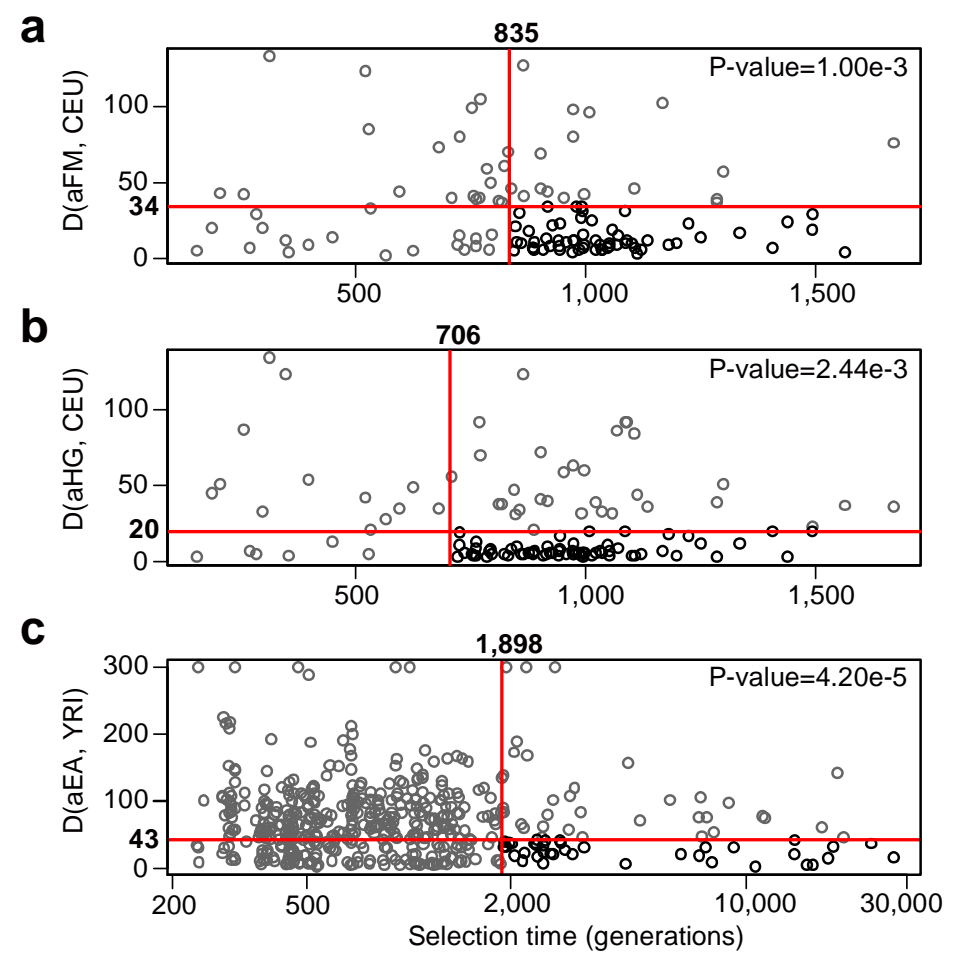

Figure 3 Estimated time of selection vs. haplotype distance from aDNA. For the PS signals, haplotype distances between aDNA and the MHG of present-day genomes $\left(D_{a H G, M H G}\right)$ were plotted against the estimated times of selection. The red crosses indicate the most significant partitions of $D_{a H G, M H G}$. The $P$ values were conducted by Chi-square test. (a) Analysis based on $D_{a H G, M H G}$ between aFM and CEU. (b) Analysis based on $D_{a H G, M H G}$ between aHG and CEU. (c) Analysis based on $D_{a H G, M H G}$ between aEA and YRI.

Based on the observed general concordance between the selection time estimates and aDNA evidences, we developed an overall chronicle of human genome adaptation that is based on PS signals, the three AMH aDNA genomes and the Neanderthal genome consensus ${ }^{40}$ (Fig. 4). The PS signals were further annotated for possible functions of evolutionary relevance (Supplementary Tables 14-16). Overall, the signals were strongly concentrated to between $0.5-1.8 \mathrm{kga}(12.5-45 \mathrm{kya})$ in CEU and $\mathrm{CHB}$, corresponding to a period of migration, population founding and agriculture. A lack of signals beyond $2 \mathrm{kga}$ (50 kya) was observed in CEU and CHB, which may be attributable to the severe bottlenecks that erased the more ancient coalescent information. In YRI, the signals stretched over a much wider time interval of 250 ga-27 kga (about 6 kya-0.7 mya), possibly due to the much weaker bottlenecks during the history of African populations. 


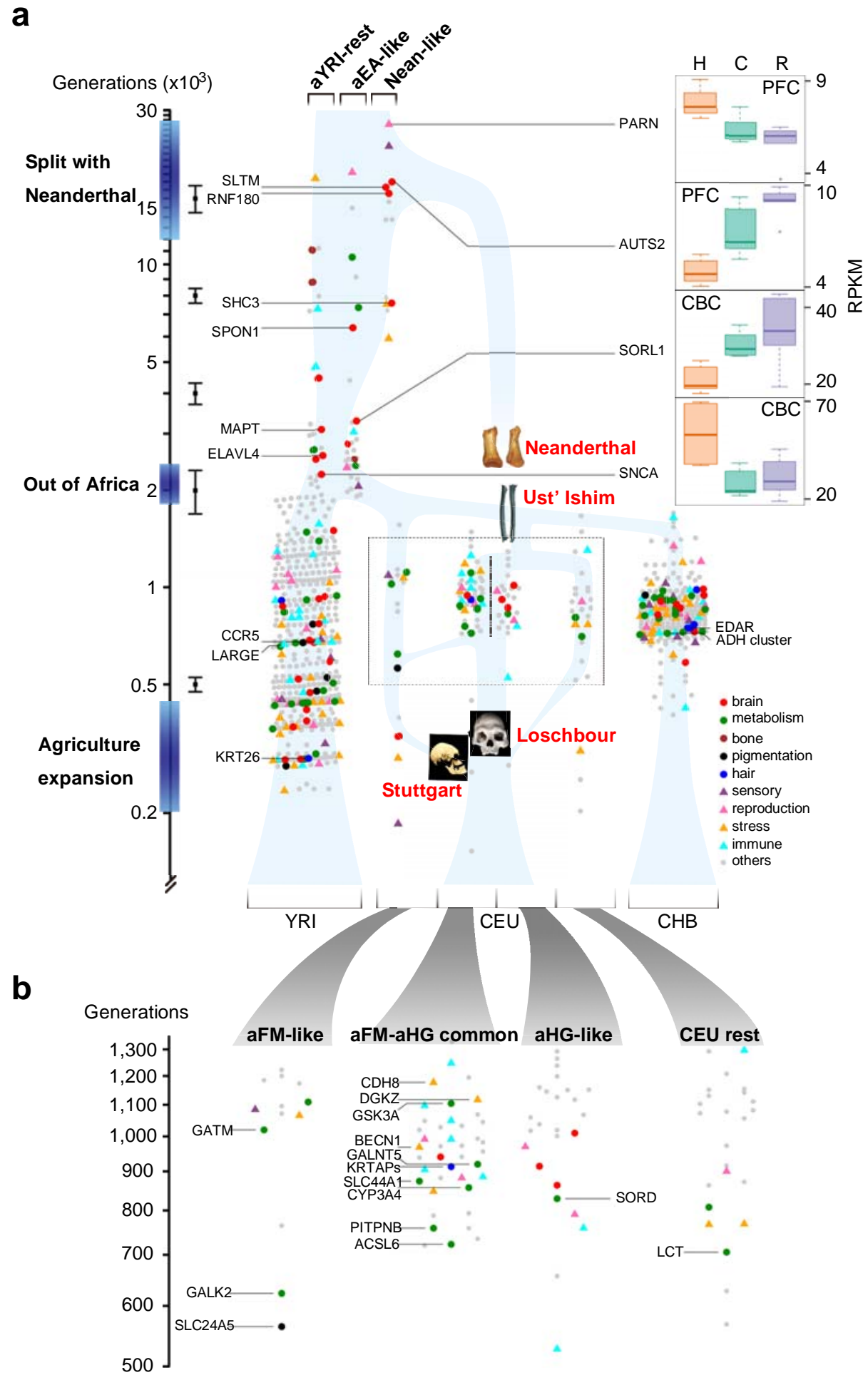

Figure 4 Timeline of PS signals in humans. Each dot represents a candidate PS signal. Genes that can be assigned to functional categories of strong relevance to human evolution were labeled in different colors and shapes (Online Methods). (a) The PS events are plotted along a bigger time scale, for all three populations. A simplified approximate population history was constructed 
based on estimated demographic trajectories and known evidences, plotted as a background graph in light blue. Error bars are standard deviations of time estimates according to simulations for 0.5 , 2, 4, 8 and $16 \mathrm{kga}$. Ancient signals ( $\geq 1,900$ generations) in YRI were classified into Nean-like, aEA-like and aYRI-rest by comparing with aEA. PARN, AUTS2, SORL1 and SNCA show human-specific expression pattern in brain regions (Supplementary Note 5). The skeleton images of the four ancient/archaic individuals were adopted from the original papers ${ }^{18,39,40}$, and placed at the assumed spatiotemporal coordinates. H: Human; C: Chimpanzee; R: Rhesus macaque; PFC: prefrontal cortex; CBC: cerebellum cortex. (b) Signals in CEU were illustrated in finer time scale for 4 groups: aFM-like, aHG-like, aFM-aHG common and CEU-rest.

One pertinent question is whether genes of various functional categories were differentially selected in different time periods. We randomly divided the past to the more recent and ancient halves, and examined whether the time division defined significant functional enrichment (Online Methods). Intriguingly, the signals older than 2,218 ga ( $\sim 55.5$ kya) were strongly enriched for brain function with an odds ratio of $7.53\left(P\right.$ value $=8.08 \times 10^{-6}$, FDR $_{\text {permut }}=0.0002$, Supplementary Fig. 11a $)$. It should be noted that this enrichment may be confounded as many ancient signals were manually annotated based on literature review (Supplementary Tables 17-19). On the other hand, the signals of stress response were significantly enriched in the recent YRI history of later than $444 \mathrm{ga}\left(\sim 11.1 \mathrm{ky}\right.$, odds ratio $=4.21, P$ value $=8.37 \times 10^{-4}$, $F_{\text {Dermut }}=0.013$, Supplementary Fig. 11b). The specific biological processes in this category include response to wounding, inflammatory response and defense response to other organism.

We defined the YRI signals older than $1.9 \mathrm{kga}$ (47.5 kya, approximately the $T_{\max }$ of aEA) as the ancient selection signals, which were further assigned to Neanderthallike (Nean-like), aEA-like, or aYRI-rest classes based on the $D_{\text {aDNA,MHG }}$ distances (Supplementary Table 20 and Online Methods). The Nean-like signals likely represent shared selection events between Neanderthal and AMH prior to their complete divergence. As shown above, a substantial fraction of genes involved in such selection events are related to brain function, AUTS2 (18,027 ga, 450 kya) and $\operatorname{SLTM}(17,355 \mathrm{ga}, \sim 434 \mathrm{kya})$ are both involved in autism spectrum disorders (ASD), affecting communication and social interaction abilities ${ }^{41,42}$. RNF180 (16,601 ga, $\sim 415 \mathrm{kya})$ regulates the brain levels of monoamine oxidase A $(M A O-A)$ and affects emotional and social behaviors via the serotonin pathway ${ }^{43}$. SHC3 (7,586 ga, 189 
kya) is almost brain-specific, highly expressed in the cerebral cortex and frontal and temporal lobes, regulates neuronal survival, and protects the CNS against environmental stresses ${ }^{44}$. Notably, these genes are all involved in the cognitive abilities of social interaction and communication.

The aEA-like signals were more closely related to aEA, and the aYRI-rest class defined all the remaining ancient signals. Interestingly, both classes harbored numerous brain function-related signals that overlapped with the emergence of AMH over time (2-8 kga or 50k-200 kya). SPON1 (6,354 ga, 159 kya) encodes a multidomain extracellular matrix protein that plays an important role in axon path-finding and early cortical development. This protein binds to the amyloid precursor protein (APP) and inhibits $\beta$-secretase cleavage of APP, which plays a central role in the pathogenesis of Alzheimer's disease (AD), wherein the uncontrolled cleavage of APP results in the accumulation of neurotoxic $\mathrm{A} \beta$ peptide $^{45}$. SORL1 $(3,285 \mathrm{ga}, \sim 82 \mathrm{kya})$ is a neuronal endocytic receptor that regulates the recycling of APP from the cell surface $^{46}$. MAPT $(3,078 \mathrm{ga}, \sim 77 \mathrm{kya})$ encodes the tau protein that assembles and stabilizes the microtubule framework of neurons. Non-specific aggregation of tau is the hallmark of $\mathrm{AD}^{47}$. ELAVL4 (2,556 ga, $\sim 64$ kya) encodes $H u D$, a neuron-specific RNA-binding protein that regulates the spatiotemporal activation of neuronal mRNAs, and affects neuronal development and plasticity, learning, and memory ${ }^{48}$. SNCA (2,234 ga, 56 kya) encodes the alpha-synuclein protein and plays an important role in the release of neurotransmitters and inter-neuronal signaling, and is also associated with $\mathrm{AD}^{49}$. Intriguingly, all these genes closely interact within a sub-network of $\mathrm{AD}$ pathogenesis (Fig. 5 and Online Methods). 


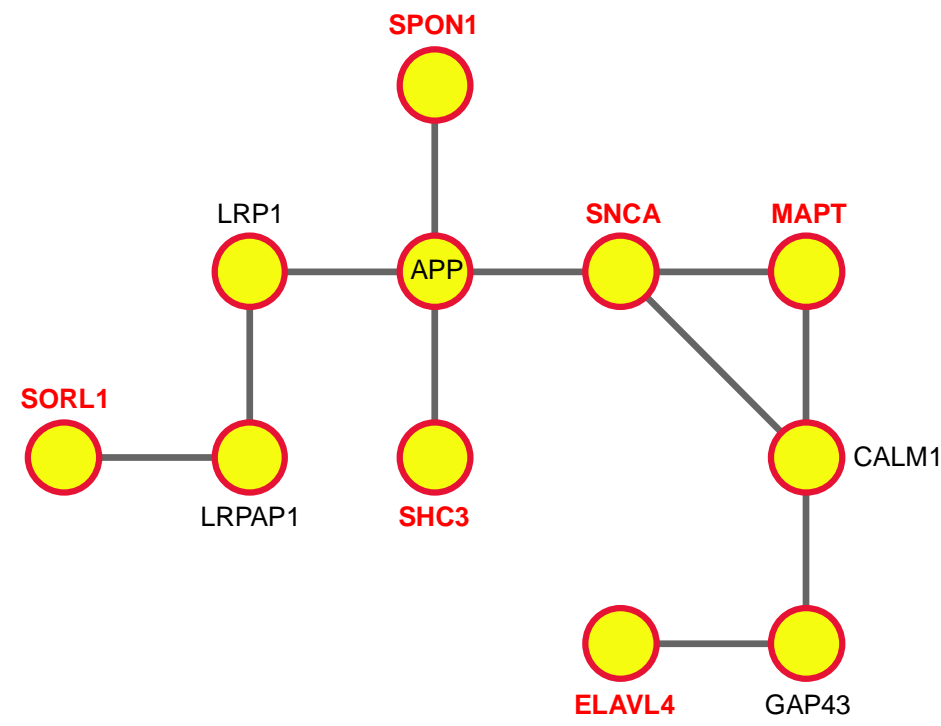

Figure 5 Protein-protein interaction sub-network of brain related genes. Genes labeled in red are candidate PS genes under ancient selection in YRI. Among the multiple routes between these genes, the shortest paths were presented.

For the recent selection events, CEU signals were divided into four classes: aFMlike, aFM-aHG common, aHG-like, and CEU-rest, depending on the $D_{\mathrm{aDNA}, \mathrm{MHG}}$ distances to aFM or aHG (Fig. 4b and Supplementary Tables 21-23, Online Methods). PS signals in the aFM-like class might have contributed to early agriculture transition. Among the aFM-like signals, GATM (1,020 ga, $\sim 25$ kya $)$ encodes glycine amidinotransferase, a key enzyme for the synthesis of creatine in the human body. Creatine is essential for energy buffering in vertebrate cells, especially in muscles, and it may also be supplied from carnivorous diets. The switch from the protein-rich Paleolithic diet to the Neolithic vegetarian-based diet might have exerted pressure on creatine self-synthesis. GALK2 is an aFM-like candidate gene (623 ga, $\sim 15.6 \mathrm{kya})$ that encodes galactokinase, which is an enzyme responsible for the conversion of galactose to glucose. SORD is another carbohydrate metabolism-related candidate gene (829 ga, $20.7 \mathrm{kya}$ ), but was found to be selected in the aHG-like class. SORD encodes sorbitol dehydrogenase, which catalyzes the conversion of sorbitol to fructose. Interestingly, galactose is primarily found in dairy food, grains, and vegetables, whereas sorbitol mainly exists in fruits. These candidate PS signals therefore seem to be congruent with the diet specificities observed in the ancestral groups. Furthermore, aFM-aHG common signals revealed numerous genes that were 
related to the response to stress or metabolism (labeled in Fig. 4b). Among these, DGKZ, SLC44A1, PITPNB, and ACSL6 are involved in lipid metabolism, and CDH8 is related to the response to cold ${ }^{50}$ and may be involved in climate adaptation.

\section{DISCUSSION}

The enrichment of PS signals in brain function beyond 55kya supports the notion that human brain has experienced rapid evolution before OOA. Surprisingly, the 5 ancient brain signals specific to $\mathrm{AMH}$ all seem to play important roles in $\mathrm{AD}$ pathogenesis. In fact, $\mathrm{AD}$ remains arguably a disease unique to humans, as full pathological evidence of $\mathrm{AD}$, particularly $\mathrm{AD}$-related neurodegeneration, are lacking in great apes ${ }^{51}$. Emerging evidence indicates that $\mathrm{AD}$ vulnerability is strongly associated with hyperconnectivity, augmented synaptic and metabolic activities, as well as functional plasticity ${ }^{52}$. We speculate that the gain of brain function during AMH emergence might have mainly affected synapse networking and neuroplasticity, and this gain was not without a price: it might have led to an increase in structural instability and regional metabolic burden that resulted in a higher risk for neurodegeneration in the aging brain. For the more recent history, the sudden increase of PS signals in stress response in YRI seems to strongly coincide with the emergence of agriculture, likely due to the expanded spectra of pathogens and parasites brought by dense dwelling, irrigation and livestock raising.

In fact, this study provided a fine resolution to the recent PS signals. Numerous well established PS signals were re-captured such as SLC24A5, LCT, EDAR, ADH gene cluster, CCR5, and $L A R G E$; and their estimated times of selection were mostly consistent with previous reports ${ }^{13,15-17}$. SLC24A5 was shown to play a pivotal role in skin pigmentation lightening in Europeans ${ }^{10}$. Interestingly, the haplotype profile of $S L C 24 A 5$ in CEU revealed a high affinity to aFM $\left(D_{a F M, M H G}=2\right)$ and a substantial distance to aHG $\left(D_{a H G, M H G}=28\right)$, as suggests that skin lightening associated with SLC24A5 originated from Near East, and likely was introduced into ancient Europeans via farming transition. This was strongly supported by a recent study based on 83 ancient DNA specimens ${ }^{53}$. Many novel candidate signals in this study may be 
worth of in-depth studies to shed lights to recent adaptations to changes in diets, climates and diseases, etc (Supplementary Tables 14-19, see Methods).

\section{METHODS}

Methods and any associated references are available in the online version of the paper.

\section{ACKNOWLEDGEMENTS}

We thank M. Wang for helpful discussion; thank D. Falush, L. Frantz and L. Tian for valuable comments. This work was supported by the Max-Planck-Gesellschaft Partner Group Grant and the National Science Foundation of China (31371267). The data presented in this paper are tabulated in the supplementary materials. The authors declare no competing financial interests.

\section{AUTHOR CONTRIBUTIONS}

K. T. and M. S. supervised the study. K. T., M. L. and L. J. designed the modeling. S. H. and R. M. implemented the modeling. K. T. and Q. F. conceived the idea of ancient genomes. Q. F. processed the ancient genomes. H. Z. did the simulations. H. Z. and J. L. analyzed the genome data. Q. Y. and P. K. designed and performed the gene expression analysis. K. T., H. Z., S. H., and M. S. wrote the manuscript with help from all co-authors.

\section{COMPETING FINANCIAL INTERESTS}

The authors declare no competing financial interests.

\section{References}

1. Nielsen, R. et al. A scan for positively selected genes in the genomes of humans and chimpanzees. PLoS Biol 3, e170 (2005).

2. Voight, B.F., Kudaravalli, S., Wen, X. \& Pritchard, J.K. A map of recent positive selection in the human genome. PLoS Biol 4, e72 (2006).

3. Wang, E.T., Kodama, G., Baldi, P. \& Moyzis, R.K. Global landscape of recent inferred Darwinian selection for Homo sapiens. Proc Natl Acad Sci U S A 103, 135-40 (2006).

4. Kelley, J.L., Madeoy, J., Calhoun, J.C., Swanson, W. \& Akey, J.M. Genomic signatures of positive selection in humans and the limits of outlier approaches. Genome Res 16, 9809 (2006).

5. Tang, K., Thornton, K.R. \& Stoneking, M. A new approach for using genome scans to detect recent positive selection in the human genome. PLoS Biol 5, e171 (2007).

6. Sabeti, P.C. et al. Genome-wide detection and characterization of positive selection in human populations. Nature 449, 913-8 (2007). 
7. Williamson, S.H. et al. Localizing recent adaptive evolution in the human genome. PLoS Genet 3, e90 (2007).

8. Grossman, S.R. et al. Identifying recent adaptations in large-scale genomic data. Cell 152, 703-13 (2013).

9. Bersaglieri, T. et al. Genetic signatures of strong recent positive selection at the lactase gene. Am J Hum Genet 74, 1111-20 (2004).

10. Lamason, R.L. et al. SLC24A5, a putative cation exchanger, affects pigmentation in zebrafish and humans. Science 310, 1782-6 (2005).

11. Miller, C.T. et al. cis-Regulatory changes in Kit ligand expression and parallel evolution of pigmentation in sticklebacks and humans. Cell 131, 1179-89 (2007).

12. Perry, G.H. et al. Diet and the evolution of human amylase gene copy number variation. Nat Genet 39, 1256-60 (2007).

13. Kamberov, Y.G. et al. Modeling recent human evolution in mice by expression of a selected EDAR variant. Cell 152, 691-702 (2013).

14. Lorenzo, F.R. et al. A genetic mechanism for Tibetan high-altitude adaptation. Nat Genet 46, 951-6 (2014).

15. Itan, Y., Powell, A., Beaumont, M.A., Burger, J. \& Thomas, M.G. The origins of lactase persistence in Europe. PLoS Comput Biol 5, e1000491 (2009).

16. Beleza, S. et al. The timing of pigmentation lightening in Europeans. Mol Biol Evol 30, 24-35 (2013).

17. Wilde, S. et al. Direct evidence for positive selection of skin, hair, and eye pigmentation in Europeans during the last 5,000 y. Proc Natl Acad Sci U S A 111, $4832-7$ (2014).

18. Lazaridis, I. et al. Ancient human genomes suggest three ancestral populations for present-day Europeans. Nature 513, 409-13 (2014).

19. Sabeti, P.C. et al. Positive natural selection in the human lineage. Science 312, 1614-20 (2006).

20. Przeworski, M. The signature of positive selection at randomly chosen loci. Genetics $\mathbf{1 6 0}$, 1179-89 (2002).

21. Hernandez, R.D. et al. Classic selective sweeps were rare in recent human evolution. Science 331, 920-4 (2011).

22. Ferrer-Admetlla, A., Liang, M., Korneliussen, T. \& Nielsen, R. On detecting incomplete soft or hard selective sweeps using haplotype structure. Mol Biol Evol 31, 1275-91 (2014).

23. Wang, M. et al. Detecting recent positive selection with high accuracy and reliability by conditional coalescent tree. Mol Biol Evol 31, 3068-80 (2014).

24. Asthana, S. et al. Widely distributed noncoding purifying selection in the human genome. Proc Natl Acad Sci U S A 104, 12410-5 (2007).

25. Chun, S. \& Fay, J.C. Identification of deleterious mutations within three human genomes. Genome Res 19, 1553-61 (2009).

26. Andres, A.M. et al. Targets of balancing selection in the human genome. Mol Biol Evol 26, 2755-64 (2009).

27. Fumagalli, M. et al. Widespread balancing selection and pathogen-driven selection at blood group antigen genes. Genome Res 19, 199-212 (2009).

28. Amambua-Ngwa, A. et al. Population genomic scan for candidate signatures of balancing selection to guide antigen characterization in malaria parasites. PLoS Genet 8, e1002992 (2012).

29. Ponting, C.P. \& Hardison, R.C. What fraction of the human genome is functional? Genome Res 21, 1769-76 (2011).

30. Lindblad-Toh, K. et al. A high-resolution map of human evolutionary constraint using 29 mammals. Nature 478, 476-82 (2011).

31. Ward, L.D. \& Kellis, M. Evidence of abundant purifying selection in humans for recently acquired regulatory functions. Science 337, 1675-8 (2012).

32. Leffler, E.M. et al. Multiple instances of ancient balancing selection shared between humans and chimpanzees. Science 339, 1578-82 (2013).

33. Rasmussen, M.D., Hubisz, M.J., Gronau, I. \& Siepel, A. Genome-wide inference of ancestral recombination graphs. PLoS Genet 10, e1004342 (2014). 
34. DeGiorgio, M., Lohmueller, K.E. \& Nielsen, R. A model-based approach for identifying signatures of ancient balancing selection in genetic data. PLoS Genet 10, e1004561 (2014).

35. Li, H. \& Durbin, R. Inference of human population history from individual wholegenome sequences. Nature 475, 493-6 (2011).

36. Abecasis, G.R. et al. An integrated map of genetic variation from 1,092 human genomes. Nature 491, 56-65 (2012).

37. Tajima, F. Statistical method for testing the neutral mutation hypothesis by DNA polymorphism. Genetics 123, 585-95 (1989).

38. Fay, J.C. \& Wu, C.I. Hitchhiking under positive Darwinian selection. Genetics 155, 1405-13 (2000).

39. $\mathrm{Fu}, \mathrm{Q}$. et al. Genome sequence of a 45,000-year-old modern human from western Siberia. Nature 514, 445-9 (2014).

40. Prufer, K. et al. The complete genome sequence of a Neanderthal from the Altai Mountains. Nature 505, 43-9 (2014).

41. Salyakina, D. et al. Variants in several genomic regions associated with asperger disorder. Autism Res 3, 303-10 (2010).

42. Oksenberg, N. \& Ahituv, N. The role of AUTS2 in neurodevelopment and human evolution. Trends Genet 29, 600-8 (2013).

43. Kabayama, M. et al. Rines E3 ubiquitin ligase regulates MAO-A levels and emotional responses. J Neurosci 33, 12940-53 (2013).

44. Troglio, F. et al. The Rai (Shc C) adaptor protein regulates the neuronal stress response and protects against cerebral ischemia. Proc Natl Acad Sci U S A 101, 15476-81 (2004).

45. Ho, A. \& Sudhof, T.C. Binding of F-spondin to amyloid-beta precursor protein: a candidate amyloid-beta precursor protein ligand that modulates amyloid-beta precursor protein cleavage. Proc Natl Acad Sci U S A 101, 2548-53 (2004).

46. Rogaeva, E. et al. The neuronal sortilin-related receptor SORL1 is genetically associated with Alzheimer disease. Nat Genet 39, 168-77 (2007).

47. Mudher, A. \& Lovestone, S. Alzheimer's disease-do tauists and baptists finally shake hands? Trends Neurosci 25, 22-6 (2002).

48. Bronicki, L.M. \& Jasmin, B.J. Emerging complexity of the HuD/ELAV14 gene; implications for neuronal development, function, and dysfunction. RNA 19, 1019-37 (2013).

49. Iwai, A. Properties of NACP/alpha-synuclein and its role in Alzheimer's disease. Biochim Biophys Acta 1502, 95-109 (2000).

50. Suzuki, S.C. et al. Cadherin-8 is required for the first relay synapses to receive functional inputs from primary sensory afferents for cold sensation. J Neurosci 27, 3466-76 (2007).

51. Finch, C.E. \& Austad, S.N. Commentary: is Alzheimer's disease uniquely human? Neurobiol Aging 36, 553-5 (2015).

52. Buckner, R.L. et al. Cortical hubs revealed by intrinsic functional connectivity: mapping, assessment of stability, and relation to Alzheimer's disease. J Neurosci 29, 1860-73 (2009).

53. Mathieson, I. et al. Eight thousand years of natural selection in Europe. BioRxiv doi: http://dx.doi.org/10.1101/016477(2015). 


\section{Online Methods}

Empirical data processing and tree construction. Only autosomal genomes from $1000 \mathrm{G}$ phase 1 were used in this study. The imputed and phased variants (ftp://ftp.1000genomes.ebi.ac.uk/vol1/ftp/release/20110521/) were first merged with the human reference genome (build 37) to generate individual haploid genome sequences. To ensure high-quality data, thorough filtering was performed on the haploid genome sequences. Briefly, the whole genome sequence was scanned with overlapping sliding $100-\mathrm{kb}$ windows and a 50-kb step size, and a window was removed when it showed $>50 \%$ sites labeled as having aberrant coverage depth or low mapping quality $^{54}$

(ftp://ftptrace.ncbi.nih.gov/1000genomes/ftp/technical/working/20120417_phase1_masks/Pilo tMask/). In total, $11.1 \%$ of the autosomal genome was removed.

Thirty pre-processed haploid genome sequences were randomly selected from each of the CEU, CHB, and YRI panels (Supplementary Table 24). To reconstruct coalescent trees, PSMC was applied to every pair of haploid genomes within each population. By default, PSMC assumes 30 fixed consecutive time intervals, where TMRCA events are to be assigned. To increase the resolution for more recent history, we allocated more time intervals in the recent and fewer in the ancient history (Supplementary Note 1.1). Such a time interval division was applied throughout this study. In total, 435 paired genomes were analyzed in each population. A unique pairwise-distance matrix was computed for every elementary consensus segment that contains no breakpoint (Supplementary Fig. 1) across all the 435 pairwise comparisons, and was used to estimate a raw coalescent tree using the UPGMA algorithm in phylip package ${ }^{55}$.

One concern with the use of $1000 \mathrm{G}$ phase 1 data is its low coverage (2-6x) sequencing. To investigate the potential impact of such low coverage, for a few individuals that have been sequenced both at low coverage in $1000 \mathrm{G}$ and high coverage in Complete Genomics ${ }^{56}$, we compared the TMRCA estimation and population inferences between the two (Supplementary Note 1.2). 
Estimation of population size trajectories from empirical data. Simulations showed that concatenated mega-sequences $(100 \times 30 \mathrm{Mb})$ resulted in reduced variance and higher accuracy in the estimation of recent population size compared to single sequences (Supplementary Fig. 12). To achieve optimal estimation, we concatenated all available diploid genomes within each $1000 \mathrm{G}$ phase 1 panel $(85,97$, and 88 individuals in CEU, CHB, and YRI, respectively) into a mega-genome and inferred the population size trajectory using PSMC (Supplementary Fig. 6).

Coalescent models and likelihood test. Assuming that coalescent trees can be directly observed and the exact demographic trajectories are given, we demonstrated that it is possible to construct a statistical model to query the evolutionary processes behind the coalescent trees. Here, we propose a coalescent-based model to try to assign each tree to the mode of neutrality, negative selection (NS), balancing selection (BS), or positive selection (PS). The model includes three hypotheses: $H_{0}, H_{1}$, and $H_{2}$, corresponding to three different coalescence patterns, as follows:

For a population of varying population size, with initial effective population size $N_{0}$ and effective population size $N_{\mathrm{e}}(t)$, assuming $n$ individuals sampled at present, Griffiths and Tavare ${ }^{57,58}$ showed that it is possible to define the rescaled coalescent time as follows:

$$
\Lambda(t)=\int_{0}^{t} \frac{d s}{\lambda(s)}
$$

where

$$
\lambda(t)=\frac{N_{\mathrm{e}}(t)}{N_{0}}
$$

indicates the scale function of population size change. Using $\tau_{i}$ to represent the rescaled coalescent waiting time from $i$ to $i-1$ ancestors, it can be shown that the coalescent process may be treated as for a standard constant population size model (Supplementary Note 2.1). Time rescaling is assumed for the rest of this section.

Under neutrality, coalescent occurs at a constant rate of $\left(\begin{array}{l}i \\ 2\end{array}\right)$ when there are $i$ ancestors. The joint density function for $\left(\tau_{n}, \tau_{n-1}, \ldots, \tau_{2}\right)$ as follows: 


$$
g\left(\tau_{n}, \tau_{n-1}, \ldots, \tau_{2}\right)=\prod_{j=2}^{n}\left(\begin{array}{l}
j \\
2
\end{array}\right) e^{-\left(\begin{array}{l}
j \\
2
\end{array}\right) \tau_{j}}
$$

This defines the $H_{0}$ model for neutrality (Supplementary Note 2.1).

When a selection event occurs, the tree pattern changes. For example, a tree that is substantially compressed indicates either PS or $\mathrm{NS}^{59}$. In contrast, a tree that strongly elongates indicates $\mathrm{BS}^{60}$. The effect of various selection processes on the coalescent trees is illustrated in Supplementary Figure 13. Following the coalescent model for neutrality, we introduce a scaling parameter $\alpha$ to describe the overall coalescent rate change. The coalescent rate therefore takes the form of $\frac{\left(\begin{array}{l}k \\ 2\end{array}\right)}{\alpha}$ for $k=n, n-1, \ldots, 2$. For neutrality, $\alpha=1$. When $\alpha>1(<1)$, the coalescent rate is smaller (larger) than the neutral rate, corresponding to accelerated (decelerated) coalescent. The joint density function of $\left(\tau_{n}, \tau_{n-1}, \ldots, \tau_{2}\right)$ is given by:

$$
g^{\prime}\left(\tau_{n}, \tau_{n-1} \ldots, \tau_{2}\right)=\prod_{j=2}^{n} \frac{\left(\begin{array}{l}
j \\
2
\end{array}\right)}{\alpha} e^{-\frac{\left(\begin{array}{l}
j \\
2
\end{array}\right)}{\alpha} \tau_{j}}
$$

which defines the $H_{1}$ model with $\alpha \neq 1$ (Supplementary Note 2.2).

Strong and recent PS cannot be distinguished from NS by $H_{1}$ alone, as both result in very small value of $\alpha$. However, PS differs from NS in having a time-dependent coalescent rate change. Based on this property, we propose a third model, $\mathrm{H}_{2}$, to distinguish the PS process. This model contains three consecutive time intervals, within which the coalescent rates remain constant (Supplementary Note 2.3). Therefore, a tree is divided into three segments by two discrete time parameters, $\tau_{i_{1}}$ and $\tau_{i_{2}}, 2<i_{1}<i_{2}<n$; the three intervals may assume different coalescent scaling coefficients of $\alpha_{1}, \alpha_{2}$, and $\alpha_{3}$.

A likelihood test framework was constructed based on the following 3 models:

$$
D_{10}=-\log \frac{\mathrm{L}\left(\operatorname{null} \mid \tau_{n}, \tau_{n-1}, \ldots, \tau_{2}\right)}{\sup _{\alpha} \mathrm{L}\left(\alpha \mid \tau_{n}, \tau_{n-1}, \ldots, \tau_{2}\right)}
$$




$$
\begin{aligned}
& D_{20}=-\log \frac{\mathrm{L}\left(\text { null } \mid \tau_{n}, \tau_{n-1}, \ldots, \tau_{2}\right)}{\sup _{\alpha_{i, i=1,2,3}} \mathrm{~L}\left(\alpha_{i, i=1,2,3}, \tau_{i_{j, j=1,2}} \mid \tau_{n}, \tau_{n-1}, \ldots, \tau_{2}\right)} \\
& D_{21}=-\log \frac{\mathrm{L}\left(\alpha \mid \tau_{n}, \tau_{n-1}, \ldots, \tau_{2}\right)}{\sup _{\alpha_{i, i=1,2,3}} \mathrm{~L}\left(\alpha_{i, i=1,2,3}, \tau_{i_{j, j=1,2}} \mid \tau_{n}, \tau_{n-1}, \ldots, \tau_{2}\right)}
\end{aligned}
$$

For each tree, the test statistics $D_{10}, D_{20}$, and $D_{21}$ are maximized, which also give rise to the estimation of the associated parameters (Supplementary Note 2). Importantly, by maximizing the likelihood of $H_{2}$, the two associated time parameters, $\tau_{i_{1}}$ and $\tau_{i_{2}}$, and the corresponding coalescent scaling coefficients, $\alpha_{1}, \alpha_{2}$, and $\alpha_{3}$, were also estimated. Depending on the patterns given by $\alpha_{1}, \alpha_{2}$, and $\alpha_{3}, \tau_{i_{1}}$, or $\tau_{i_{2}}$ or nearby time points were searched for the best pattern that matches the initiation of PS selection, and hence to estimate the selection starting time $\tau_{s}$. The details of estimation of selection time and coefficient can be found in Supplementary Note 2.4 and 2.5, respectively. Finally, $\tau_{s}$ is mapped back to the absolute generation time scale as $t_{s}$, by applying a reverse function of Equation 1.

Rescaling and correction of the raw trees. The raw trees estimated from empirical data were first rescaled to the coalescent time scale according to Equation 1, by using the demographic trajectories estimated from the mega-genomes. Successful detection of selection relies on proper tree construction and rescaling; UPGMA and PSMC may both introduce estimation errors during this process. We designed a systematic correction to the rescaled trees to minimize such errors. It assumes that the overall genome coalescent profile is neutral and therefore should fit the $H_{0}$ distribution. Under neutrality and assuming exact tree inference, the estimator of coalescent scaling coefficient $\alpha(\alpha *)$ follows $\Gamma\left(n-1, \frac{1}{n-1}\right)$, with a median approximately at 1 . Given the estimated waiting times in a tree as $\tau^{\prime}=\left(\tau_{n}^{\prime}, \tau_{n-1}^{\prime}, \ldots, \tau_{2}^{\prime}\right)$, we first controlled the overall $\alpha *$ by dividing all $\tau^{\prime}$ against the genome-wide median of $\alpha_{*}$. Furthermore, within each coalescent interval, $\tau_{i}$ should follow an exponential distribution with median $\ln 2 /\left(\begin{array}{l}i \\ 2\end{array}\right)$. We normalized $\tau_{i}^{\prime}$ by dividing it by $m_{i}\left(\begin{array}{l}i \\ 2\end{array}\right) / \ln 2$, where $m_{i}$ is the median for $\tau_{i}^{\prime}$. The efficacy of correction was evaluated in simulations on 10 models 
of population size change over time (Supplementary Fig. 14 and Supplementary Note 3.1). Statistics including $\alpha_{*}, D_{10}, D_{20}$ and $D_{21}$ were calculated either from the trees reconstructed by PSMC and UPGMA with correction, or directly from the corresponding true coalescent trees. Results show that the types of estimates were highly consistent either between the reconstructed and true trees (Supplementary Fig. 4), or for the true trees for the different demographic scenarios (Supplementary Fig. $15)$.

Annotate signals of selection in empirical data. The genome-wide rescaled trees were scanned for candidate BS, NS, or PS signals. A tree is called a BS signal when the coalescent scaling coefficient $\alpha>1$ and $D_{10}>D_{10}^{b}$, where $D_{10}^{b}$ is the threshold for BS. When a tree satisfies $\alpha<1$ and $D_{10}>D_{10}^{p n}$, it is called as a NS/PS signal, which can be NS or PS depending on the consequential evaluation of the $H_{2}$-related criteria. $D_{10}^{b}$ is the threshold to reject neutrality for $\alpha<1 . D_{10}^{b}$ and $D_{10}^{p n}$ were obtained by a reshuffling procedure across empirical genome-wide trees. In brief, it is known that a tree in the rescaled time is defined by the series of coalescent waiting times $\left(\tau_{n}, \tau_{n-1}, \ldots\right.$, $\tau_{2}$ ), and tree topology is nuisance information in this study. Under neutrality, $\tau_{i}$ and $\tau_{j}$ are independent, given $i \neq j$. Therefore $\tau_{i}$ is exchangeable across different trees for the same $i$ th interval, without affecting the global neutral distribution. Random reshuffling was performed as earlier described for all the intervals across all the trees. The reshuffled tree set was divided into 2 halves with $\alpha<1$ and $\alpha \geq 1$, and $D_{10}$ was calculated separately in each half to give the corresponding null distribution for NS and BS, respectively. Afterwards, the upper $0.1 \%$ quantile in the $D_{10}$ for BS was assigned $D_{10}^{b}$; and the upper $1 \%$ quantile of $D_{10}$ for NS was used to define $D_{10}^{p n}$, respectively (Supplementary Table 25). A more stringent threshold was applied to BS due to an excessive upper tail in the empirical distribution.

Based on $\mathrm{H}_{2}$, we constructed two different tests for PS signatures of either recent or ancient events. A recent PS is expected to affect both temporal $\left(H_{2}\right.$ signature $)$ and global coalescent rates ( $H_{1}$ signature). Therefore, for the recent PS signals, we propose a test (RPS test) based on both $D_{10}$ and $D_{21}$. A tree is called a PS signal when $\alpha<1, D_{10}>D_{10}^{p n}$, and $D_{21}>D_{21}^{r p}\left(\right.$ called NS when $\left.D_{21}>D_{21}^{r p}\right) \cdot D_{21}^{r p}$ was obtained by reshuffling the trees that satisfy $\alpha<1$ and $D_{10}>D_{10}^{p n}$. The upper $20 \%$ quantile of the 
$D_{21}$ distribution from this reshuffled tree set was designated as $D_{21}^{r p}$. On the other hand, when the PS events were more ancient, the whole tree compression was not so evident, but the time dependence of the coalescent rate persisted; therefore, the test for ancient PS signals (APS) requires $\alpha<1$ and $D_{21}>D_{21}^{a p} \cdot D_{21}^{a p}$ was derived by reshuffling the tree subset that satisfies $\alpha<1$ and $D_{10} \leq D_{10}^{p n}$. The upper $1 \%$ quantile of $D_{21}$ from this reshuffled tree set was designated as $D_{21}^{a p}$ (Supplementary Table 25).

For BS and PS, candidate tree signals (SG-tree) were combined to define candidate regions. For BS signals, neighboring SG-trees were concatenated to define the BS regions (Supplementary Tables 7-9). For each PS test, SG-trees located within $100 \mathrm{~kb}$ from each other were concatenated to define candidate regions. In view of extensive LD in genome regions affected by recent positive selection ${ }^{2,5}$, RPS candidate regions shorter than $100 \mathrm{~kb}$ were excluded from further analysis. For the APS test, very short candidate regions $(<20 \mathrm{~kb}$ ) or regions defined by single SG-trees were also omitted. Supplementary Tables 1-3 list the candidate regions of PS in 3 continental populations.

Since hitchhiking during a selective sweep results in progressively decreasing diversity toward the selection center, we used the SG-tree of maximum $D_{10}$ in a candidate region to define the center of a PS. The distribution of distance between the estimated and true centers of selection is shown in Supplementary Figure 16. For each PS candidate region, the regional estimate of selection coefficient, $s_{\text {reg }}$, takes the median value of all trees in that region, $s_{\text {reg }}=\operatorname{median}\left(s_{\text {tree, }} i\right)$. Analogously, the regional selection time, $t_{\text {reg }}$, was estimated as median $\left(t_{\text {tree, }}{ }_{i}\right)$. Noticing an obvious underestimation of selection starting time for ancient PS (Supplementary Fig. 17), we made a simple correction to $t_{\text {reg }}$ : if $t_{\text {reg }}>1500, t_{r e g}^{\prime}=t_{\text {reg }}+2 \times\left(t_{\text {reg }}-1500\right)$; if $t_{\text {reg }} \leq$ $1500, t^{\prime}{ }_{r e g}=t_{\text {reg. }} . s_{\text {reg }}$, and $t^{\prime}{ }_{\text {reg }}$ were used for estimation of empirical data. The corrected time estimation was thoroughly evaluated in simulations (Supplementary Fig. 1e-1g and Supplementary Fig. 8) and applied on empirical data. The major statistics were plotted for several candidate PS regions from simulations (Supplementary Fig. 18) and 1000G data (Supplementary Fig. 19). 
Power tests. For the simulated cases of BS and NS, neutral simulations were generated by using the same demographic models, and the neutral $D_{10}$ distribution was calculated to define the cutoffs at a level of $0.5 \%$. BS and NS tests were then conducted in the simulated cases of selection as for the empirical data, to estimate the test power. Supplementary Figure 5 shows the power to detect BS and NS.

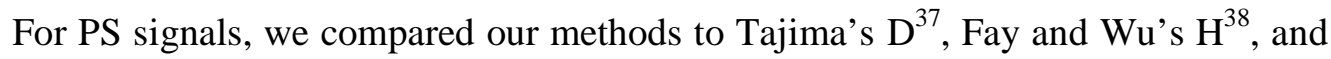
iHS $^{2}$. Tajima's D and Fay and Wu's H were calculated for non-overlapping 50-SNP windows. PS and neutral simulations were generated for the same sets of demographic models. iHS was calculated for SNPs with minor allele frequency (MAF) $\geq 0.05$. In a 50-SNP window, the number of SNPs with $|\mathrm{iHS}| \geq 2$ was used as the test statistic. RPS and APS tests follow the procedures as for the empirical data.

In case of a hard sweep, we first examined the test power in three realistic demographic trajectories corresponding to those estimated for CEU (Supplementary Fig. 1c), CHB (Supplementary Fig. 20a), and YRI (Fig. 1a) from 1000G data. For all tests, 30 haplotypes were sampled and 1,500 cases of PS were randomly simulated at a uniform density, for a selection starting time range between present-day to 2,500 ga, and a selection coefficient range from 0.01 to 0.2 . For the YRI-like model, we also simulated ancient selection events, at a grid of selection starting time of 2,000, 4,000, $8,000,16,000$, and 24,000 ga, with fixed selection coefficient values of 0.005, 0.01, $0.02,0.05,0.1$, and 0.2 , respectively (Fig. 1b). The neutral simulations estimated the false positive rates of RPS and APS to be $2 \%$ and $3.5 \%$, respectively. The cutoffs of the other three statistics (D and H scores and iHS) were set to assume the same false positive rate of $2 \%$. We also evaluated the test power using a constant size demography assuming a $N_{\mathrm{e}}$ of 10,000 . In this scenario, the sample sizes for Tajima's D, Fay and Wu's H, and iHS tests were set at 120, which would render better performance for tests sensitive to small sample sizes such as iHS. The sample sizes for RPS and APS tests were kept at 30. The resulting power for hard sweeps for each test was plotted in Figure 1a-c and Supplementary Figure 20a,b.

For selection on standing variation (a soft sweep), power comparison was conducted under a core model of demography (see Supplementary Fig. 21 and section 
on Simulations below), with the sample sizes all set to 30 . We also performed a comparison in the constant population size model as for the hard sweep. All procedures followed those for the hard sweep. The results are plotted in Figure 1d and Supplementary Figure 20c.

Simulations. For all simulations in this study, a constant mutation rate of $2.5 \times 10^{-8}$ $\mathrm{bp}^{-1}$.generation ${ }^{-1}$ and a constant recombination rate of $1.3 \times 10^{-8} \mathrm{bp}^{-1}$. generation $^{-1}$ were assumed. Supplementary Note 3 specifies all the details of the model parameters.

Essential neutral data was first simulated for the three realistic demographic trajectories by $\mathrm{MSMS}^{61}$. In addition, a core model was simulated, that assumes a simplified demography, but consisted of common features of human populations such as bottlenecks and a recent expansion (Supplementary Fig. 21). A total of 100 replicates of 100-Mb sequences were simulated for each demography model.

MSMS was also used for all the PS simulations. A total of 100 replicates of $2-\mathrm{Mb}$ sequences were simulated under each designated demography and selection parameter set. Selection was assumed to act on a novel mutation at the center of each sequence. The times of selection were set from 1 to 2,500 ga for both hard and soft sweeps. For hard sweep, the selection coefficient of the advantageous allele assumed a uniform sampling between 0.01 and 0.2 . For soft sweep, a constant selection coefficient of 0.05 was used, and the initial frequencies of advantageous alleles ranged from 0.01 to 0.1 .

We used SFS_CODE ${ }^{62}$ to simulate NS by assigning a variable proportion of mutations as being under selection. We set the proportion of non-synonymous mutations to $10 \%, 50 \%$, and $100 \%$, and the selection coefficient to $0.001,0.01$, and 0.1 . A total of 100 replicates of $200-\mathrm{kb}$ sequences were simulated for each parameter set.

Increased genetic diversity that results in more deeply structured coalescent trees is the signature of BS. To test the power to detect BS, we simulated an increased density of polymorphic sites by elevating the mutation rate. We set a wide range of fold 
changes of the basic mutation rate: $1.2,1.5,2,3,5$, and 10. Soft sweep, NS, and BS were all simulated under both the constant-size model and the core model.

Functional annotation and enrichment test. For PS and BS, any protein-coding genes (Ensembl, www.ensembl.org) that overlapped with the candidate regions were defined as candidate genes. The enrichment of PS and BS candidate genes was tested by DAVID ${ }^{63,64}$ (http://david.abcc.ncifcrf.gov/) using unmasked protein-coding genes as the background gene list. Numerous categories were examined, including functional category (SP_PIR_KEYWORDS), gene ontology (GOTERM_BP_FAT, GOTERM_CC_FAT, GOTERM_MF_FAT, PANTHER_BP_ALL and PANTHER_MF_ALL), pathways (KEGG_PATHWAY and PANTHER_PATHWAY) and tissue expression (GNF_U133A_QUARTILE, UNIGENE_EST_QUARTILE, and UP_TISSUE). Terms with $P$ value $<0.05$ after Benjamini correction were significantly enriched. Supplementary Tables 4-6 show the enriched terms for PS genes in 3 populations, while Supplementary Tables 11-13 show enriched terms for BS genes in 3 populations. For BS, we also conducted enrichment analysis in YRI using the PANTHER web tool ${ }^{65}$, which uses a binomial test (with Bonferroni correction for multiple testing) to calculate the significance of enrichment (Supplementary Table 10).

We further classified the candidate PS genes into several highly specific functional categories based on the relevant GO terms ${ }^{66}$, including brain development, food metabolism, bone morphology, pigmentation, hair, sensory perception, reproduction, response to stress, and immune process, which was further curated by knowledge and text mining (Supplementary Tables 17-19). In Figure 4, only the functional categories of the center genes were presented. If a center gene belongs to multiple categories, then the category of higher functional relevance (manually curated) was used (Supplementary Tables 14-16).

Temporal enrichment analysis. To test whether there is any time-dependent enrichment of PS signals of certain functions, we split the selection events into the ancient and recent periods using a sliding time division. Fisher's exact test was used to see if there is any heterogeneity of gene functions between the recent and ancient PS signals. To test a particular functional category, we treated the PS signals of the 
category of interest as "cases" and all the other PS signals as "controls". 10,000 permutations were then performed by randomly reshuffling the time of selection events to calculate the false discovery rate (FDR).

Ancient DNA analyses. We used the same genotype calls for Ust'-Ishim, aFM, and aHG as in previous studies ${ }^{18,39}$. Sites included for analysis were required to have a minimum root-mean-square mapping quality of $\mathrm{MQ} \geq 30$. For the Neanderthal sequence, we removed all sites with genotype quality $<40$, and mapping quality $<30$, as done previously ${ }^{67}$.

We clustered present-day human haplotypes (PHs) into haplotype groups if the mutual nucleotide distance was $<1$. The major haplotype group (MHG) was defined as the haplotype group with the highest frequency. For each candidate PS region, the haplotype distance measurement is conducted for a 50-kb window around the estimated selection center. The haplotype distance between the MHG and the ancient haplotypes (aDNA) was defined as the mean of the pairwise distances between aDNA and each PH within MHG:

$$
D_{a D N A, M H G}=\operatorname{mean}\left(\sum_{i=1}^{n} D_{a D N A, P H_{i}}\right)
$$

where $D_{a D N A, P H}$ is the pairwise differences between two unphased ancient haplotypes and the $\mathrm{PH}^{68}$.

For the calculation of $T_{\max D}$, we split the haplotype distance/time space into 4 quadrants, and used a Chi-square test to search possible combinations for the most significant division, conditional on an enrichment of small $D_{a D N A, M H G}$ in the ancient part (e.g., right lower quadrant in Fig. 3a). As the Chi-square test is sensitive to small observed values, we used the constraint that no more than one quadrant has data points $\leq 10$. A total of 1,000 permutations were then performed by randomly reshuffling the time of selection among PS signals to calculate the false discovery rate.

To assign CEU signals into different classes, we used the following criteria:

1. aFM-like, $D_{a F M, M H G} \leq 20 \& D_{a H G, M H G}>20$;

2. aHG-like, $D_{a H G, M H G} \leq 20 \& D_{a F M, M H G}>20$;

3. aFM-aHG common, $D_{a F M, M H G} \leq 20 \& D_{a H G, M H G} \leq 20$; 
4. CEU-rest, others.

Supplementary Tables 21-23 show the first 3 classes of PS in CEU. Similarly, to assign the ancient YRI signals into different classes, the following conditions were applied:

1. Nean-like, $D_{\text {Nean, } M H G} \leq 50 \& T \geq 5,000$ generations;

2. aEA-like, $D_{a E A, M H G} \leq 50$;

3. aYRI-rest, others.

Supplementary Table 20 shows the classified PS signals in YRI.

Network analysis. SPON1, MAPT, SNCA, SORL1, SHC3, and ELAVL4 were mapped to a protein-protein interaction (PPI) network ${ }^{69}$ (Figure 5). Cytoscape $^{70}$ v3.2.0 was used to plot the sub-network connected by shortest paths, centered on amyloid precursor protein (APP).

\section{References}

54. Abecasis, G.R. et al. A map of human genome variation from population-scale sequencing. Nature 467, 1061-73 (2010).

55. Felsenstein, J. PHYLIP - Phylogeny Inference Package (Version 3.2). Cladistics 5, 164166 (1989).

56. Drmanac, R. et al. Human genome sequencing using unchained base reads on selfassembling DNA nanoarrays. Science 327, 78-81 (2010).

57. Griffiths, R.C. \& Tavare, S. Sampling theory for neutral alleles in a varying environment. Philos Trans R Soc Lond B Biol Sci 344, 403-10 (1994).

58. Griffiths, R.C. \& Tavaré, S. The ages of mutations in gene trees. Ann Appl Prob 9, 567590 (1999).

59. Kaplan, N.L., Hudson, R.R. \& Langley, C.H. The "hitchhiking effect" revisited. Genetics 123, 887-99 (1989).

60. Hudson, R.R. \& Kaplan, N.L. The coalescent process in models with selection and recombination. Genetics 120, 831-40 (1988).

61. Ewing, G. \& Hermisson, J. MSMS: a coalescent simulation program including recombination, demographic structure and selection at a single locus. Bioinformatics 26, 2064-5 (2010).

62. Hernandez, R.D. A flexible forward simulator for populations subject to selection and demography. Bioinformatics 24, 2786-7 (2008).

63. Huang, D.W., Sherman, B.T. \& Lempicki, R.A. Systematic and integrative analysis of large gene lists using DAVID bioinformatics resources. Nat Protoc 4, 44-57 (2009).

64. Huang, D.W., Sherman, B.T. \& Lempicki, R.A. Bioinformatics enrichment tools: paths toward the comprehensive functional analysis of large gene lists. Nucleic Acids Res 37, 113 (2009).

65. Mi, H., Muruganujan, A., Casagrande, J.T. \& Thomas, P.D. Large-scale gene function analysis with the PANTHER classification system. Nat Protoc 8, 1551-66 (2013).

66. Ashburner, M. et al. Gene ontology: tool for the unification of biology. The Gene Ontology Consortium. Nat Genet 25, 25-9 (2000).

67. Meyer, M. et al. A high-coverage genome sequence from an archaic Denisovan individual. Science 338, 222-6 (2012). 
68. Huerta-Sanchez, E. et al. Altitude adaptation in Tibetans caused by introgression of Denisovan-like DNA. Nature 512, 194-7 (2014).

69. Keshava Prasad, T.S. et al. Human Protein Reference Database--2009 update. Nucleic Acids Res 37, D767-72 (2009).

70. Shannon, P. et al. Cytoscape: a software environment for integrated models of biomolecular interaction networks. Genome Res 13, 2498-504 (2003). 